\title{
Retrospective analysis of intensity-modulated radiotherapy and three-dimensional conformal radiotherapy of postoperative treatment for biliary tract cancer
}

Hyo Chun Lee ${ }^{1}$, Jong Hoon Lee ${ }^{1}$, Sea-Won Lee ${ }^{2}$, Joo Hwan Lee ${ }^{1}$ Mina Yư ${ }^{3}$ Hong Seok Jang ${ }^{2}$, Sung Hwan Kim

'Department of Radiation Oncology, St. Vincent's Hospital, College of Medicine, The Catholic University of Korea, Suwon, Korea ${ }^{2}$ Department of Radiation Oncology, Seoul St. Mary's Hospital, College of Medicine, The Catholic University of Korea, Seoul, Korea ${ }^{3}$ Department of Radiation Oncology, Buchoen St. Mary's Hospital, College of Medicine, The Catholic University of Korea, Bucheon, Korea

Purpose: This study was conducted to compare the outcome of three-dimensional conformal radiotherapy (3D-CRT) and intensity-modulated radiotherapy (IMRT) for the postoperative treatment of biliary tract cancer.

Materials and Methods: From February 2008 to June 2016, 57 patients of biliary tract cancer treated with curative surgery followed by postoperative 3D-CRT ( $n=27)$ or IMRT $(n=30)$ were retrospectively enrolled.

Results: Median follow-up time was 23.6 months (range, 5.2 to 97.6 months) for all patients and 38.4 months (range, 27.0 to 89.2 months) for survivors. Two-year recurrence-free survival is higher in IMRT arm than 3D-CRT arm with a marginal significance (25.9\% vs. $47.4 \% ; p=0.088)$. Locoregional recurrence-free survival $(64.3 \%$ vs. $81.7 \% ; p=0.122)$ and distant metastasis-free survival $(40.3 \%$ vs. $55.8 \% ; p=0.234$ ) at two years did not show any statistical difference between two radiation modalities. In the multivariate analysis, extrahepatic cholangiocarcinoma, poorly-differentiated histologic grade, and higher stage were significant poor prognostic factors for survival. Severe treatment-related toxicity was not significantly different between two arms.

Conclusions: IMRT showed comparable results with 3D-CRT in terms of recurrence, and survival, and radiotherapy toxicity for the postoperative treatment of biliary tract cancer.

Keywords: Biliary tract neoplasms, Radiotherapy, Adjuvant, Survival, Toxicity

\section{Introduction}

Biliary tract cancer, which consists of cholangiocarcinoma and gallbladder cancer, is well known for its poor prognosis. The reported 5-year overall survival rate is around 5\%-30\% [1-5]. Surgical excision is the treatment of choice for all detectable biliary tract cancer, but complete resection rates are not high enough to achieve cure and recurrence following surgery is common, either at local or distant sites [6-8]. Accordingly, adjuvant treatment is recommended following surgical management, although optimal strategy is not clearly determined [9]. Recently, intensity-modulated radiotherapy (IMRT) has become an alternative to threedimensional conformal radiotherapy (3D-CRT) in upper

Received 22 July 2019, Revised 25 November 2019, Accepted 18 December 2019.

Correspondence: Sung Hwan Kim, Department of Radiation Oncology, St. Vincent's Hospital, 93 Jungbu-daero, Paldal-gu, Suwon 16247, Korea. Tel: +82-31-249-7560, Fax: +82-31-257-3734, E-mail: kimandre@catholic.ac.kr (http://orcid.org/00000002-0139-5003)

(c) This is an Open Access article distributed under the terms of the Creative Commons Attribution Non-Commercial License (http://creativecommons.org/ licenses/by-nc/4.0/) which permits unrestricted non-commercial use, distribution, and reproduction in any medium, provided the original work is properly cited.

www.e-roj.org 
gastrointestinal cancers, such as pancreas and periampullary cancer for its ability to decrease the treatment related toxicity while successfully achieve dose escalation [10]. Similar to pancreas cancer, biliary tract cancer is also surrounded by number of critical organs and subsequent treatment related toxicity is severe. There are trials of adjuvant radiotherapy utilizing 3D-CRT and IMRT, but endpoints did not focus on the survival and/or toxicity of the two modalities $[11,12]$. In the multicenter retrospective studies, long-term local control was achieved with higher radiation dose ( $\geq 50-54$ Gy), without significant difference in acute adverse events in postoperative radiotherapy of biliary tract cancer, and this might encourage the extended use of IMRT for its feasibility of dose escalation $[13,14]$. To the best of our knowledge, there was no study which directly compared the results of postoperative treatment of resectable biliary tract cancer in terms of radiotherapy technique, in this case, 3D-CRT versus IMRT. Therefore, the purpose of this study is to compare the outcome of 3D-CRT and IMRT in postoperative treatment of biliary tract cancer.

\section{Materials and Methods}

Between February 2008 and April 2016, collectively 57 biliary tract cancer patients whose medical records assembled from three medical institutions were reviewed retrospectively. This study was approved by the Institutional Review Board of The Catholic University of Korea Catholic Medical Center (No. VC18RESI0014). The eligibility criteria were: (1) histologically confirmed bile duct or gallbladder adenocarcinoma; (2) received curative surgery followed by adjuvant radiotherapy within 60 days; (3) planned and completed at least 45 Gy of postoperative radiotherapy; (4) no evidence of distant metastasis.

Clinical staging work-up included physical examination, complete blood count, blood chemistry for evaluation of jaundice and liver function, level of tumor marker CA19-9, chest and abdominal computed tomography (CT), and positron emission tomography/computed tomography (PET/CT) along with magnetic resonance image (MRI) of the abdomen if feasible. The 8th edition of the American Joint Committee on Cancer staging system was used for both clinical and pathological staging.

\section{Treatment}

All patients were treated with curative surgery. Surgical procedure was selected based on tumor site and extent, listed as follows: 18 liver lobectomy with bile duct resection, 11 pylorus-preserving pancreaticoduodenectomy, 10 bile duct resection, 16 cholecystectomy, 1 extended hepatectomy with bile duct resection, and 1 common bile duct total segmental resection with Roux-en-Y hepaticojejunostomy. The interval between surgery and radiotherapy ranged from 18 to 60 days, median 39 days. Contrast-enhanced simulation CT was acquired to generate the radiotherapy planning. Radiotherapy was planned using 3D-CRT techniques in 27 (47.4\%) patients, and IMRT planning techniques in 30 (52.6\%) patients.

In 2 of 3 institutions, all 16 patients were treated with 3D-CRT plans. The other institution used both planning method for 21 patients until May 2014. Twenty patients enlisted since August 2014 were treated with IMRT. The differences between institutions and treatment period were not statistically significant.

For 3D-CRT plans, target was delineated based on the postoperative tumor bed and draining lymph nodes. An 1-cm margin was added to make up for set-up errors. A 3or 4-field box beam arrangement was used with anteriorposterior, posterior-anterior, and right and left lateral beams. For IMRT, the clinical target volume (CTV) was generated which encompassed the postoperative tumor bed and draining lymph nodes. The planning target volume (PTV) was expanded from CTV by 0.5 to $1 \mathrm{~cm}$ to every dimension, accounting for set-up error and organ movement.

The 3D-CRT was delivered with 6-15 MV photons using the linear accelerator. TomoTherapy (Accuray Inc., Sunnyvale, CA, USA) was utilized to deliver IMRT plan. Vac-Lock and wing board were used to ensure the immobilization of patients in IMRT and 3D-CRT, respectively. At least $45 \mathrm{~Gy}$ of radiation dose was prescribed, and up to 56.9 Gy was delivered depending on initial tumor extent, gross or microscopic residual diseases, and relationship with surrounding organs at risk (OAR). Dosevolume histograms were produced for the PTV and all the OARs. The optimization was constrained to deliver greater than 95\% of the prescription dose to the PTV. Quantitative Analysis of Normal Tissue Effects in Clinics (OUANTEC) parameters were used to evaluated the dosimetric doses to OAR. Concurrent chemotherapy was utilized in all but one patient, due to poor general condition. The 5-fluorouracil (5-FU) was the most commonly used regimen, 400 to $600 \mathrm{mg} / \mathrm{m}^{2}$ injected intravenously once a week during the radiotherapy. Adjuvant chemotherapy was delivered to 13 patients, using FL (5-FU and leucovorin) or FP (5-FU and cisplatin) regimen.

\section{Statistical analyses}

The primary endpoint was to determine overall survival (OS), recurrence-free survival (RFS), locoregional recurrence-free survival (LRFS), and distant metastasis-free survival (DMFS), of 
Table 1. Patients' characteristics

\begin{tabular}{|c|c|c|c|}
\hline Characteristic & $3 D-C R T(n=27)$ & IMRT $(n=30)$ & $p$-value \\
\hline Age at diagnosis (yr) & $62.5 \pm 8.2$ & $61.4 \pm 9.4$ & 0.889 \\
\hline Sex & & & 0.462 \\
\hline Male & $17(63.0)$ & $16(53.3)$ & \\
\hline Female & $10(37.0)$ & $14(46.7)$ & \\
\hline Tumor site & & & 0.083 \\
\hline Intrahepatic cholangiocarcinoma & $15(55.6)$ & $8(26.7)$ & \\
\hline Extrahepatic cholangiocarcinoma & $6(22.2)$ & $12(40.0)$ & \\
\hline Gallbladder carcinoma & $6(22.2)$ & 10 (33.3) & \\
\hline Presence of jaundice at diagnosis & & & 0.400 \\
\hline No & $17(63.0)$ & $22(73.3)$ & \\
\hline Yes & $10(37.0)$ & $8(26.7)$ & \\
\hline Histologic grade & & & 0.642 \\
\hline Well differentiated & $4(14.8)$ & $6(20.0)$ & \\
\hline Moderately differentiated & $19(70.4)$ & $17(56.7)$ & \\
\hline Poorly differentiated & $4(14.8)$ & $7(23.3)$ & \\
\hline Residual disease & & & 0.160 \\
\hline R0 resection & $15(55.6)$ & 22 (73.3) & \\
\hline $\mathrm{R} 1$ resection & $12(44.4)$ & $8(26.7)$ & \\
\hline Lymphovascular invasion & & & 1.000 \\
\hline No & $10(40.0)$ & $12(40.0)$ & \\
\hline Yes & $15(60.0)$ & $18(40.0)$ & \\
\hline Perineural invasion & & & 0.097 \\
\hline No & $7(28.0)$ & $15(50.0)$ & \\
\hline Yes & $18(72.0)$ & $15(50.0)$ & \\
\hline Stage & & & 0.451 \\
\hline I & $2(7.4)$ & $1(3.3)$ & \\
\hline$\|$ & $9(33.3)$ & $13(43.3)$ & \\
\hline III & $14(51.9)$ & 16 (53.3) & \\
\hline IV & $2(7.4)$ & $0(0.0)$ & \\
\hline Adjuvant chemotherapy & & & 0.025 \\
\hline No & $17(63.0)$ & $27(90.0)$ & \\
\hline Yes & $10(37.0)$ & $3(10.0)$ & \\
\hline Radiotherapy interval (day) & $39.7 \pm 10.0$ & $32.8 \pm 10.1$ & 0.008 \\
\hline Radiation dose (Gy) & $50.6 \pm 2.8$ & $50.3 \pm 3.5$ & 0.237 \\
\hline
\end{tabular}

Values are presented as mean \pm standard deviation or number (\%). 3D-CRT, three-dimensional conformal radiotherapy; IMRT, intensity-modulated radiotherapy.

3D-CRT and IMRT. Pearson chi-square test was used to analyze the distribution of variables. Kaplan-Meier method was used to calculate the actuarial OS, RFS, LRFS, and DMFS rates. OS was defined as the time from operation to date of death or the last day of follow-up. RFS was defined as the time from operation to date of disease progression, death, or the last day of follow-up. LRFS and DMFS were defined as the time from operation to date of locoregional recurrence and distant metastasis, respectively. Univariate and multivariate analysis was performed for prognostic factors using the log-rank test and Cox proportional hazards model. p-values less than 0.05 were considered statistically significant. Statistical analysis was performed with R version 3.2.1 (http://www.R-project.org).

\section{Results}

Patient and tumor characteristics are shown in Table 1. The median age of all patients at the time of diagnosis was 60 years, ranging from 47 to 79 years. Age, sex, pathologic stage, histologic grade, residual disease, and total radiation dose were distributed between 3D-CRT and IMRT groups with no significant difference. The mean interval between surgery and radiotherapy differed significantly between groups, 39.7 days in 3D-CRT and 32.8 days in IMRT group ( $p=0.013$ ).

\section{Recurrence and survival}

The median follow-up time for all patients was 23.6 months (range, 5.2 to 97.6 months). As for the survivors, median 
follow-up time was 38.4 months (range, 27.0 to 89.2 months). Median OS time for all patients, 3D-CRT, and IMRT group were

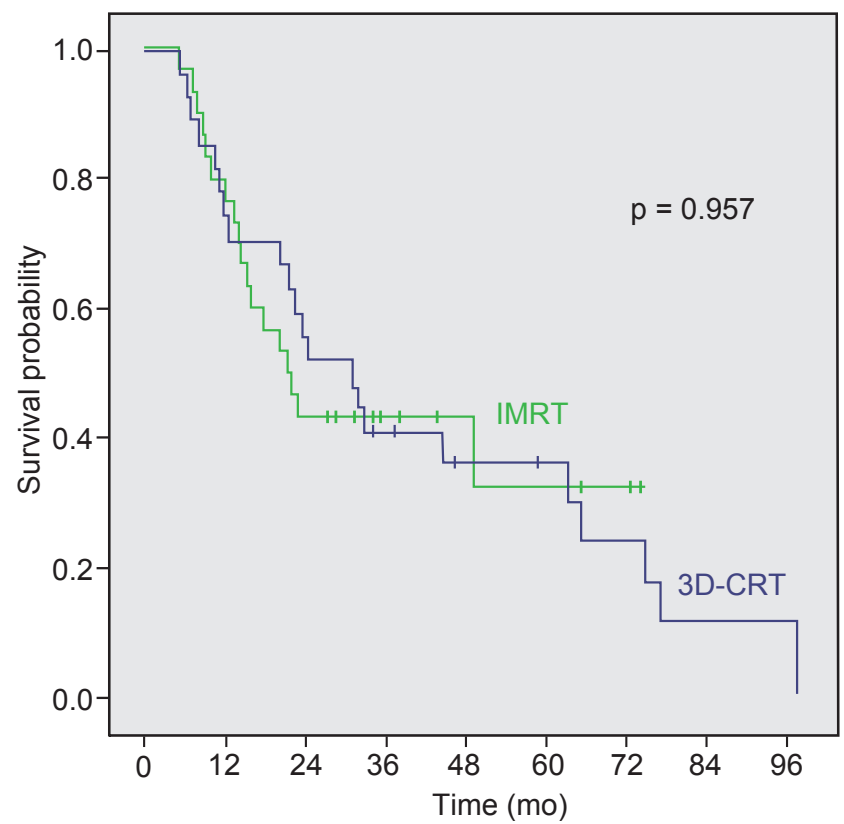

Fig. 1. Kaplan-Meier overall survival curves of intensitymodulated radiotherapy (IMRT) arm and three-dimensional conformal radiotherapy (3D-CRT) arms.
23.6, 31.3, and 21.7 months, respectively. The 3-year OS rates were $40.7 \%$ in 3D-CRT group and $43.3 \%$ in IMRT group ( $p=$

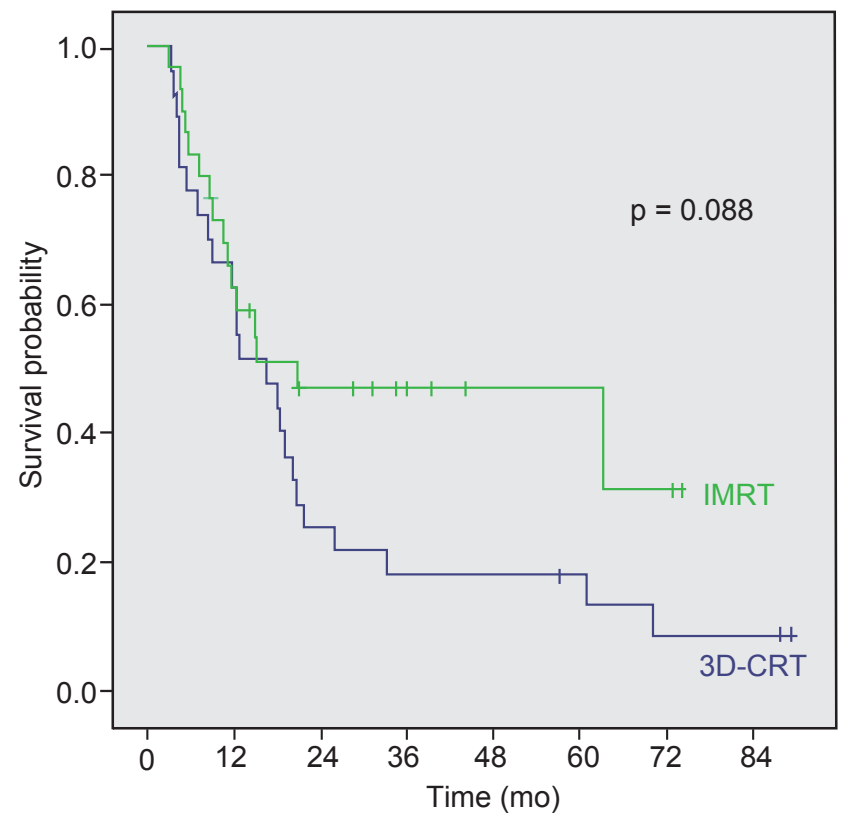

Fig. 2. Kaplan-Meier recurrence-free survival curves of intensitymodulated radiotherapy (IMRT) arm and three-dimensional conformal radiotherapy (3D-CRT) arms.

Table 2. Analyses of factors associated with overall survival

\begin{tabular}{|c|c|c|c|c|}
\hline & \multicolumn{2}{|c|}{ Univariate } & \multicolumn{2}{|l|}{ Multivariate } \\
\hline & $\mathrm{HR}(95 \% \mathrm{Cl})$ & $p$-value & $\mathrm{HR}(95 \% \mathrm{Cl})$ & $\mathrm{p}$-value \\
\hline Age (yr) & & 0.019 & & 0.086 \\
\hline$\leq 60$ & 1 & & 1 & \\
\hline$>60$ & $2.164(1.136-4.123)$ & & $0.497(0.224-1.104)$ & \\
\hline Tumor site & & 0.076 & & - \\
\hline Intrahepatic & 1 & & - & \\
\hline Extrahepatic & 0.969 (0.484-1.939) & 0.929 & - & \\
\hline Gallbladder & $0.359(0.143-0.901)$ & 0.029 & - & \\
\hline Tumor grade & & 0.068 & & 0.024 \\
\hline Well differentiated & 1 & & 1 & \\
\hline Moderate differentiated & $0.771(0.328-1.815)$ & 0.552 & $0.443(0.175-1.120)$ & 0.085 \\
\hline Poorly differentiated & $2.031(0.754-5.468)$ & 0.161 & $1.438(0.524-3.946)$ & 0.480 \\
\hline Lymphovascular invasion & & 0.033 & & - \\
\hline No & 1 & & - & \\
\hline Yes & $2.099(1.060-4.156)$ & & - & \\
\hline Perineural invasion & & 0.003 & & 0.001 \\
\hline No & 1 & & 1 & \\
\hline Yes & $3.132(1.490-6.582)$ & & 3.761 (1.695-8.345) & \\
\hline Stage & & 0.093 & & 0.023 \\
\hline । & 1 & & 1 & \\
\hline$\|$ & $1.944(0.432-8.750)$ & 0.386 & $1.262(0.152-10.450)$ & 0.829 \\
\hline III & $2.033(0.407-8.855)$ & 0.345 & $1.435(0.186-11.056)$ & 0.729 \\
\hline IV & 117.735 (1.512-91.091) & 0.019 & $16.529(1.320-207.008)$ & 0.030 \\
\hline
\end{tabular}

$\mathrm{HR}$, hazard ratio; $\mathrm{Cl}$, confidence interval. 
Table 3. Analyses of factors associated with recurrence-free survival

\begin{tabular}{|c|c|c|c|c|}
\hline & \multicolumn{2}{|c|}{ Univariate } & \multicolumn{2}{|l|}{ Multivariate } \\
\hline & $\mathrm{HR}(95 \% \mathrm{Cl})$ & p-value & HR (95\% Cl) & p-value \\
\hline Radiotherapy modality & & 0.093 & & - \\
\hline 3D-CRT & 1 & & - & \\
\hline IMRT & $0.581(0.308-1.096)$ & & - & \\
\hline Age (yr) & & 0.022 & & 0.019 \\
\hline$\leq 60$ & 1 & & 1 & \\
\hline$>60$ & $2.089(1.111-3.927)$ & & $2.347(1.147-4.800)$ & \\
\hline Tumor site & & 0.003 & & 0.072 \\
\hline Intrahepatic & 1 & & 1 & \\
\hline Extrahepatic & $0.690(0.344-1.384)$ & 0.296 & $0.425(0.189-0.957)$ & 0.039 \\
\hline Gallbladder & $0.242(0.097-0.607)$ & 0.002 & $0.513(0.167-1.577)$ & 0.244 \\
\hline Tumor grade & & 0.032 & & 0.019 \\
\hline Well differentiated & 1 & & 1 & \\
\hline Moderate differentiated & 0.779 (0.334-1.018) & 0.564 & $1.137(0.419-3.084)$ & 0.801 \\
\hline Poorly differentiated & $2.384(0.890-6.388)$ & 0.084 & 4.339 (1.322-14.239) & 0.016 \\
\hline Lymphovascular invasion & & 0.025 & & - \\
\hline No & 1 & & - & \\
\hline Yes & $2.208(1.106-4.406)$ & & - & \\
\hline Perineural invasion & & 0.004 & & 0.02 \\
\hline No & 1 & & 1 & \\
\hline Yes & $2.885(1.416-5.877)$ & & 2.968 (1.190-7.401) & \\
\hline Jaundice & & 0.059 & & 0.06 \\
\hline No & 1 & & 1 & \\
\hline Yes & $1.860(0.916-3.543)$ & & $2.149(0.970-4.764)$ & \\
\hline
\end{tabular}

$\mathrm{HR}$, hazard ratio; $\mathrm{Cl}$, confidence interval; 3D-CRT, three-dimensional conformal radiotherapy; IMRT, intensity-modulated radiotherapy.

0.957) (Fig. 1). Two-year RFS (25.9\% vs. 47.4\%; $p=0.088)$, LRFS (64.3\% vs. 81.7\%; $p=0.122)$, and DMFS (40.3\% vs. 55.8\%; $p=$ $0.234)$ rates showed no statistical difference between 3D-CRT and IMRT arms (Fig. 2).

\section{Risk factors for recurrence and survival}

The prognostic factors of OS, RFS, LRFS, and DMFS in the univariate analysis are shown in Table 2. Age older than 60 and poor histologic grade were factors associated with poor outcome in the OS and RFS. Intrahepatic cancer presented better RFS compare to other types of cholangiocarcinoma ( $p$ $=0.043$ ). The radiotherapy modality was not a significant risk factor of survival in the univariate analysis. In the multivariate analysis, tumor site, histology, and stage were significant prognostic factors for OS. Other factors associated with RFS, LRFS, and DMFS are listed in Table 3.

\section{Toxicity}

Toxicity was assessed with the Common Terminology Criteria for Adverse Events v4.0. Grade 3 and 4 hematologic toxicities occurred in 9 patients, 5 in 3D-CRT and 4 in IMRT group, respectively. There was a case of grade 3 liver abscess in IMRT group, which occurred 8 months after radiotherapy. All other cases of toxicity were reported during the radiotherapy or within 1 month of completion. A patient in 3D-CRT group was hospitalized with grade 3 sepsis 1 month after the treatment while the other one in IMRT group complained of grade 3 nausea and vomiting during the adjuvant radiotherapy.

\section{Discussion and Conclusion}

The standard adjuvant therapy of biliary tract cancer is not established due to low incidence of disease entity and resultant lack of prospective randomized controlled trial. Even retrospective studies directly comparing the treatment result of IMRT with conventional 3D-CRT are occasionally reported. In a retrospective study which studied 38 cases of intrahepatic null-margin resection cholangiocarcinoma, 14 patients were treated with IMRT. Median OS of IMRT group was 21.8 months compared to 5.0 month of surgery-only group $(p=0.049)$, and DMFS were 12.5 and 5.5 months, respectively, in both groups ( $p=0.081)$ [15]. The study result suggested that adjuvant IMRT is safe and effective treatment option following surgery. However, survival analysis regarding radiotherapy technique was not assessed. There were two studies which analyzed dosimetric comparison of IMRT and 3D-CRT, one in resected 


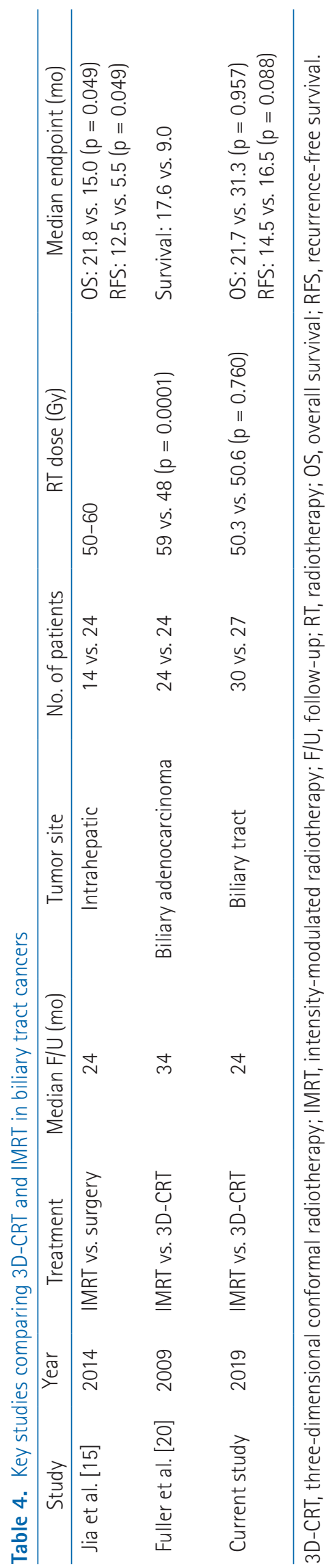

gallbladder cancer [16] and the other in pancreas and bile duct cancer which included unresectable cases [17]. The mean dose of PTV and OARs such as right kidney, liver, and small bowel were significantly decreased in IMRT plans, but survival and toxicity analyses were not reported between treatment groups. Since the advantage of IMRT is dose constraint on OARs, a few studies focused on toxicity were found, although mainly in pancreas cancer instead of biliary tract cancer. Yovino et al. [18] reported that IMRT significantly improved grade 3 and 4 acute gastrointestinal toxicity such as nausea/ vomiting and diarrhea in pancreatic and ampullary cancers compared to 451 3D-CRT cohort of RTOG 97-01. Likewise, in the report of 205 locally advanced pancreatic cancer including unresectable cases, grade 2 or higher gastrointestinal toxicity occurred less in IMRT group, even though the median radian dose was significantly higher than 3D-CRT [19]. A study with biliary tract cancer survival analysis was reported by Fuller et al. [20], compared 24 IMRT and 24 3D-CRT patients, including $14(29 \%)$ unresectable cases. The median survival was 17.6 and 9.0 months, respectively in IMRT and 3D-CRT groups, although statistical significance was not stated. Grade 2 or higher gastrointestinal toxicity was also lower in IMRT group, even with higher mean radiation dose (Table 4).

In this study, 30 IMRT cases and 27 3D-CRT cases were compared after surgical resection of biliary tract cancer. Radiation dose between two groups were not different (50.3 Gy of IMRT vs. 50.6 Gy of 3D-CRT). The 3-year OS did not show statistical difference $(p=0.957)$ and the RFS showed the tendency of favoring IMRT ( $p=0.088)$, although still not statistically significant. The treatment volume was smaller in IMRT owing to reduced PTV margin, yet drew the similar results in survival and toxicity compared with 3D-CRT.

Limitations of this study should be acknowledged. First of all, the retrospective nature of this study weakens the authority of clinical implication. The rarity of disease entity itself and low resectability caused by initially advanced disease status make it difficult to conduct a prospective randomized controlled trial. Even though this study comprised the medical records from three different territorial institutions, the number of patient was not large enough to analyze by the subsites of cholangiocarcinoma and gallbladder cancer separately. In addition, resectable cases regardless of pathologic stage were included, causing further heterogeneity of patient population. The uneven patient distribution between 3D-CRT and IMRT group could also have affected the result. Finally, the follow-up duration was relatively short to assess the late recurrences and complications.

Nevertheless, the value of this study still stands being the 
first report of comparison between 3D-CRT and IMRT in biliary tract cancer solely in adjuvant setting, to the best of our knowledge. According to our data, IMRT did not show superior result over 3D-CRT. However, IMRT has proven to be a safe and effective modality of postoperative treatment in biliary tract cancer that is non-inferior to 3D-CRT. Since IMRT is a relatively newer modality in biliary tract cancer radiotherapy and being more commonly used in clinics, a prospective randomized controlled trial is expected to clarify the role of adjuvant radiotherapy with IMRT.

\section{Conflict of Interest}

No potential conflict of interest relevant to this article was reported.

\section{Acknowledgments}

The authors wish to acknowledge the financial support of the St. Vincent's hospital, research institute of medical science (No. SVHR-2019-08). This work was supported by a grant from the National RetD Program for the Ministry of Science and ICT, Republic of Korea (No. 2017R1C1B5015762).

\section{References}

1. Siegel $R$, Naishadham D, Jemal A. Cancer statistics, 2012. CA Cancer J Clin 2012;62:10-29.

2. Nagorney DM, Kendrick ML. Hepatic resection in the treatment of hilar cholangiocarcinoma. Adv Surg 2006;40:159-71.

3. de Jong MC, Nathan H, Sotiropoulos GC, et al. Intrahepatic cholangiocarcinoma: an international multi-institutional analysis of prognostic factors and lymph node assessment. J Clin Oncol 2011;29:3140-5.

4. Zaydfudim VM, Rosen $C B$, Nagorney DM. Hilar cholangiocarcinoma. Surg Oncol Clin N Am 2014;23:247-63.

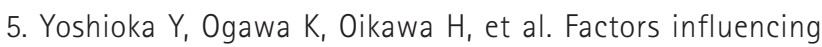
survival outcome for radiotherapy for biliary tract cancer: a multicenter retrospective study. Radiother Oncol 2014;110:546-52.

6. Hasegawa S, Ikai I, Fujii H, Hatano E, Shimahara Y. Surgical resection of hilar cholangiocarcinoma: analysis of survival and postoperative complications. World J Surg 2007;31:1256-63.

7. Kim MY, Kim JH, Kim Y, Byun SJ. Postoperative radiotherapy appeared to improve the disease free survival rate of patients with extrahepatic bile duct cancer at high risk of loco-regional recurrence. Radiat Oncol J 2016;34:297-304.
8. Koo TR, Eom KY, Kim IA, et al. Patterns of failure and prognostic factors in resected extrahepatic bile duct cancer: implication for adjuvant radiotherapy. Radiat Oncol J 2014;32:63-9.

9. Cereda S, Belli C, Reni M. Adjuvant treatment in biliary tract cancer: to treat or not to treat? World J Gastroenterol 2012;18:2591-6.

10. Taremi M, Ringash J, Dawson LA. Upper abdominal malignancies: intensity-modulated radiation therapy. Front Radiat Ther Oncol 2007;40:272-88.

11. Wang SJ, Lemieux A, Kalpathy-Cramer J, et al. Nomogram for predicting the benefit of adjuvant chemoradiotherapy for resected gallbladder cancer. J Clin Oncol 2011;29:4627-32.

12. Ben-Josef E, Guthrie KA, El-Khoueiry AB, et al. SWOG S0809: a phase II intergroup trial of adjuvant capecitabine and gemcitabine followed by radiotherapy and concurrent capecitabine in extrahepatic cholangiocarcinoma and gallbladder carcinoma. J Clin Oncol 2015;33:2617-22.

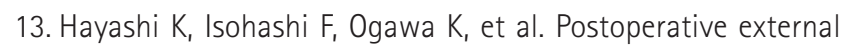
irradiation of patients with primary biliary tract cancer: a multicenter retrospective study. Anticancer Res 2015;35:6231-7.

14. Im JH, Seong J, Lee J, et al. Postoperative radiotherapy dose correlates with locoregional control in patients with extrahepatic bile duct cancer. Radiat Oncol J 2014;32:7-13.

15. Jia AY, Wu JX, Zhao YT, et al. Intensity-modulated radiotherapy following null-margin resection is associated with improved survival in the treatment of intrahepatic cholangiocarcinoma. J Gastrointest Oncol 2015;6:126-33.

16. Sun XN, Wang 0 , Gu BX, et al. Adjuvant radiotherapy for gallbladder cancer: a dosimetric comparison of conformal radiotherapy and intensity-modulated radiotherapy. World J Gastroenterol 2011;17:397-402.

17. Milano MT, Chmura SJ, Garofalo MC, et al. Intensitymodulated radiotherapy in treatment of pancreatic and bile duct malignancies: toxicity and clinical outcome. Int J Radiat Oncol Biol Phys 2004;59:445-53.

18. Yovino S, Poppe $M$, Jabbour $S$, et al. Intensity-modulated radiation therapy significantly improves acute gastrointestinal toxicity in pancreatic and ampullary cancers. Int J Radiat Oncol Biol Phys 2011;79:158-62.

19. Prasad $S$, Cambridge $L$, Huguet $F$, et al. Intensity modulated radiation therapy reduces gastrointestinal toxicity in locally advanced pancreas cancer. Pract Radiat Oncol 2016;6:78-85.

20. Fuller CD, Dang ND, Wang SJ, et al. Image-guided intensity-modulated radiotherapy (IG-IMRT) for biliary adenocarcinomas: initial clinical results. Radiother Oncol 2009;92:249-54. 